\title{
Generalization of the Momentum-Space Subtraction Procedure for Renormalized Perturbation Theory
}

\author{
M. Gomes ${ }^{\star}$ \\ Instituto de Física, Universidade de São Paulo, São Paulo, Brazil \\ J. H. Lowenstein ${ }^{\star \star}$ and W. Zimmermann ${ }^{\star \star}$ \\ Department of Physics, New York University, New York, N. Y., USA
}

Received July 1, 1974

\begin{abstract}
The momentum-space subtraction procedure for defining renormalized Feynman integrals is modified to allow for subtraction operators more general than the usual Taylor operators. The added generality permits one to assign subtraction degree less than four to some terms of the unperturbed Lagrangian.
\end{abstract}

\section{Introduction}

In the momentum-space version $[1,2]$ of $\mathrm{BPH}$ renormalized perturbation theory $[3,4]$, there now exist standard techniques $[5,6]$ for deriving field equations and Ward-Takahashi identities for composite fields (such as currents). The methods of calculation are straightforward once one has written down a Lagrangian density $\mathscr{L}_{\text {EFF }}$ as a linear combination of field products of canonical dimension less than or equal to four (more generally, the space-time dimension), and assigned to each such product $M$ a subtraction degree $\delta(M)$, with $\operatorname{dim} M \leqq \delta(M) \leqq 4$. There is one rather awkward feature of this procedure, namely the constraint, inherent in the subtraction scheme of Ref. [1], that all terms of the unperturbed part $\mathscr{L}_{0}$ of $\mathscr{L}_{\text {EFF }}$ must be assigned degree four. There are numerous applications, most notably where masses are associated with spontaneous symmetry breaking, where it is desirable to assign some terms of $\mathscr{L}_{0}$ a degree less than four. In the present note we modify the subtraction procedure and convergence proof of Ref. [1] so as to permit "soft" (degree less than four) terms in $\mathscr{L}_{0}$.

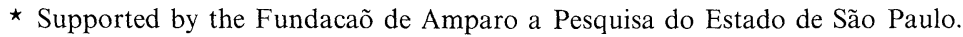

$\star \star$ Supported in part by the National Science Foundation Grants Nos. GP36316X, GP $36481 \mathrm{X}$, and GU 3186. 


\section{The Subtracted Integrand}

In renormalized perturbation theory, Green's functions are expressed, via the Gell-Mann-Low formula, as sums of contributions from various Feynman diagrams. Each diagram corresponds, in momentum space, to a product of delta functions of energy-momentum conservation, free two-point functions and integrals of the type

$$
\int\left(I_{G}(p, k)-\text { subtractions }\right) d k
$$

where $G$ is a proper (amputated, one-particle irreducible) subdiagram of $\Gamma$, with external momenta $p=\left\{p_{1}, \ldots p_{n}\right\}$ and internal "loop" momenta $k=\left\{k_{1}, \ldots k_{m}\right\}$. Typically $I_{G}$ has the form

$$
I_{G}=\prod_{a} P_{a}\left(l_{a \tau}\right) \prod_{b c \sigma} \triangle_{b c \sigma}\left(l_{b c \sigma}\right)
$$

where $P_{a}$ is a polynomial in the momenta $l_{a \tau}$ flowing into the vertex $V_{a}$, and $\triangle_{a b \sigma}$ is a function of the momentum $l_{a b \sigma}$ flowing through the line $L_{a b \sigma}$ connecting vertices $V_{a}$ and $V_{b}$. It is of the form

$$
\triangle_{a b \sigma}\left(l_{a b \sigma}\right)=\frac{P_{a b \sigma}\left(l_{a b \sigma}\right)}{l_{a b \sigma}^{2}-\mu_{a b \sigma}^{2}+i 0}
$$

where $P_{a b \sigma}$ is a polynomial in $l_{a b \sigma}^{\mu}, \mu=0,1,2,3$, and the mass parameters $m_{1}, m_{2}, \ldots$, of the theory and $\mu_{a b \sigma}^{2}$ is a non-negative, homogeneous (of degree 2) function of the mass parameters alone (further restrictions on $\mu_{a b \sigma}^{2}$ will be introduced below). In order to accommodate models in which the unperturbed propagators have poles at more than one value of $p^{2}$, we do not exclude the possibility $L_{a b \varrho}=L_{a b \sigma}$ for $\varrho \neq \sigma$.

As in Ref. [1], the subtraction procedure consists of a set of rules for constructing a "renormalized" integrand $R_{G}(p, k, m, w, \varepsilon)$, where we have made explicit the dependence on $m=m_{1}, \ldots, m_{d}$ and $w=w_{1}, \ldots, w_{e}$, the "hard" and "soft" mass parameters of the theory, respectively. For $\varepsilon>0$, one must verify that

$$
J_{G \varepsilon}=\int d k R_{G}(p, k, m, w, \varepsilon)
$$

is absolutely convergent and, for $\varepsilon \rightarrow 0$, the integral tends to a covariant tempered distribution. Specifically we define

where

$$
R_{G}=S_{G} \sum_{U \in \mathscr{F}_{G}} \prod_{\gamma \in U}\left(-\tau_{\gamma} S_{\gamma}\right) I_{G}(U)
$$

(a) $\mathscr{F}_{G}$ is the set of all forests (families of non-trivial, non-overlapping, one-particle irreducible subdiagram) of $G$. 
(b) $I_{G}(U)$ is the unsubtracted integrand written as a function of variables associated with $U$. In particular

$$
\begin{aligned}
\triangle_{a b \sigma}\left(l_{a b \sigma}\right) & \rightarrow \frac{i P_{a b \sigma}\left(l_{a b \sigma}^{\gamma}, m, w^{\gamma}\right)}{l_{a b \sigma}^{\gamma 2}-\mu_{a b \sigma}^{2}\left(m, w^{\gamma}\right)+i \varepsilon\left(l_{a b \sigma}^{\gamma 2}+\mu_{a b \sigma}^{2}\right)} \\
P_{a}\left(l_{a \tau}\right) & \rightarrow P_{a}\left(l_{a \tau}^{\gamma}, m, w^{\gamma}\right),
\end{aligned}
$$

where $\gamma$ is the smallest element of $U$ containing $L_{a b \sigma}$ (resp. $V_{a}$ ) and

$$
l_{a b \sigma}^{\gamma}=q_{a b \sigma}^{\gamma}\left(p^{\gamma}\right)+k_{a b \sigma}^{\gamma}\left(k^{\gamma}\right)
$$

where $p^{\gamma}=$ external momenta of $\gamma$, considered as independent variables, $k^{\gamma}=$ "loop" momenta of $\gamma$, which, if the routing of momentum through subdiagrams is done in an admissible fashion (see Ref. [1] for a precise definition), are linear combinations of the $k_{i}^{S}$ for $\delta \in U, \gamma \subset \delta$.

(c) $S_{\gamma}$ is the substitution operator defined by

$$
\begin{aligned}
& {\left[S_{\gamma} f\right]\left(p^{\gamma}, k^{\gamma}, m, w^{\gamma}\right)=f\left(p^{\beta}\left(p^{\gamma}, k^{\gamma}\right), k^{\beta}\left(k^{\gamma}\right), m, w^{\gamma}\right)} \\
& \quad \text { for } \beta, \gamma \in U, \quad \beta \subset \gamma,
\end{aligned}
$$

with the functional relation on the righthand side of (6) determined by energy-momentum conservation at the external vertices of $\beta$.

(d) $\tau_{\gamma}$ is the subtraction operator for the subgraph $\gamma$, defined in the next section. In most applications $\tau_{\gamma}$ will be the Taylor series $t_{p^{\gamma} w^{\gamma}}^{d(\gamma)}$ to order $d(\gamma)$, the superficial divergence of $\gamma$, in the variables $p^{\gamma}$ and $w^{\gamma}$, taken about $p^{\gamma}=w^{\gamma}=0$.

(e) The factors in the product $\Pi_{\gamma \in U}$ are written such that if $\gamma \subset \delta$, $\left(-\tau_{\gamma} S_{\gamma}\right)$ stands to the right of $\left(-\tau_{\delta} S_{\delta}\right)$.

\section{Subtraction Operators}

To specify what we mean by a subtraction operator, we consider the class $\mathscr{Y}$ of functions of the following form:

$$
F=\frac{A}{C}
$$

where $A$ and $C$ are homogeneous polynomials in three sets of fourmomentum variables, $p=\left\{p_{1} \ldots p_{a}\right\}, t=\left\{t_{1} \ldots t_{b}\right\}$ and $u=\left\{u_{1}, \ldots, u_{c}\right\}$, and two sets of mass variables, $m=\left\{m_{1}, \ldots, m_{d}\right\}$ and $w=\left\{w_{1} \ldots w_{e}\right\}$, with $C$ factorizable as

$$
C=\prod_{\alpha=1}^{n}\left(l_{\alpha}^{2}+\mu_{\alpha}^{2}+i \varepsilon\left(l_{\alpha}^{2}+\mu_{\alpha}^{2}\right)\right)
$$


where $l_{\alpha}=\Sigma \alpha_{i} p_{i}+\Sigma \beta_{j} t_{j}+\Sigma \gamma_{k} u_{k}$, with $\Sigma \beta_{j} t_{j} \equiv 0$ only if $\Sigma \alpha_{i} p_{i} \equiv 0$, and $\mu_{\alpha}^{2}$ is a non-negative, homogeneous function of degree two in $m$ and $w$. The functions $\mu_{\alpha}^{2}$ need not be polynomials in the mass variables, but they must be consistent with the polynomial nature of $C^{1}$.

We now list a number of properties of the Taylor operator $t_{p w}^{d}$ applied to functions of class $\mathscr{Y}$ :

(i) If $F \in \mathscr{Y}$, then $t_{p w}^{d} F \in \mathscr{Y}$.

(ii) $t_{p w}^{d}$ is a linear mapping which is independent of the choice of basis for the external momenta.

(iii) $t_{p w}^{d} F$ is a polynomial in $p$ and $w$ of degree $\leqq d$.

(iv) For $p=\lambda p_{0}, w=\lambda w_{0}, \lambda \rightarrow 0, \lambda^{-(d+1)}\left(1-t_{p w}^{d}\right) F \rightarrow$ const.

(v) $\operatorname{deg}_{t p w} t_{p w}^{d} F \leqq \operatorname{deg}_{t p w} F$.

(vi) $\operatorname{deg}_{t} t_{p w}^{d} F \leqq \operatorname{deg}_{t} F$.

(vii) $\operatorname{deg}_{t}\left(1-t_{p w}^{d}\right) F \leqq \operatorname{deg}_{t p w} F-d-1$.

Property (i) is trivially verified, and (ii)-(iv) are well known properties of the Taylor series to order $d$ and its remainder. The proof of the remaining properties is completely straightforward. To show the role of the special form of the function $F$, we verify explicitly inequality (vii), leaving the remaining two inequalities as an exercise for the reader.

Proof of (vii):

First, we write

$$
F=\frac{A}{C}=\frac{\sum_{i} A_{i} B_{i}}{D E}
$$

where $B_{i}$ is a monomial in $t, p$ and $w$,

$$
E=\prod_{\alpha \in I_{E}}\left(l_{\alpha}^{2}-\mu_{\alpha}^{2}+i \varepsilon\left(l_{\alpha}^{2}+\mu_{\alpha}^{2}\right)\right)
$$

where

$$
l_{\alpha}=\Sigma a_{\alpha_{l}} t_{i}+\Sigma b_{\alpha j} p_{j}+\Sigma c_{\alpha k} u_{k}
$$

with $\Sigma_{i} a_{\alpha i} t_{i} \neq 0$ for all $\alpha \in I_{\mathrm{E}} \subset\{1,2, \ldots, n\}$, and $A_{i}$ and $D$ are independent of $t, p, w$.

To establish (vii), it will be sufficient to verify this property for each $F_{i}=\frac{B_{i}}{E}$.

\footnotetext{
${ }^{1}$ Recall that $C$ may contain more than one factor with the same momentum $l$. Suppose that $l_{\alpha}=l$ for $\alpha=1, \ldots, n$ and suppose that $\mu_{\alpha}^{2}$ are the real roots of a polynomial of degree $n$ whose coefficients are polynomials in the masses; then $\mu_{\alpha}^{2}$ could very well be irrational functions of the masses, while $\Pi_{\alpha=1}^{n}\left(l^{2}-\mu_{\alpha}^{2}+i \varepsilon\left(l^{2}+\mu_{\alpha}^{2}\right)\right)$ would necessarily be a polynomial in all variables. This situation in fact arises in the explicitly broken Higgs model [8].
} 
Property (iv) and the homogeneity of $F_{i}$, imply

$$
\operatorname{deg}_{t u m}\left(1-t_{p w}^{d}\right) F_{i} \leqq \operatorname{deg} F_{i}-d-1 .
$$

But $\left(1-t_{p w}^{d}\right) F_{i}$ has the form $\frac{R(t u p m w)}{E(t u p m w) E(t u 0 m 0)^{d+1}}$ where $R$ is a homogeneous polynomial: Using the fact that $\operatorname{deg}_{t} E=\operatorname{deg}_{t p w} E=\operatorname{deg} E$, we have

$$
\operatorname{deg}_{t}\left(1-t_{p w}^{d}\right) F_{i} \leqq \operatorname{deg}_{t u m}\left(1-t_{p w}^{d}\right) F_{i}
$$

and $\operatorname{deg} F_{i}=\operatorname{deg} B_{i}-\operatorname{deg} E=\operatorname{deg}_{t p w} B_{i}-\operatorname{deg}_{t} E=\operatorname{deg}_{t p w} F_{i}$, so that Eq. (9) implies property (vii).

In a given field theoretical model, the number of distinct mass terms $\mu_{\alpha}^{2}$ which can appear in expression (8) is severely limited and we may restrict our attention to a subset $\mathscr{X}$ of $\mathscr{Y}$. We define a subtraction operator for the model under consideration to be any mapping $\tau_{p w}^{d}$ from $\mathscr{X}$ into $\mathscr{X}$ satisfying requirements (ii)-(vii) above.

\section{Modified Convergence Proof}

To prove the absolute convergence of $J_{G \varepsilon}$, we shall need the powercounting theorem of Ref. [1], suitably generalized to allow for the general subtraction operators introduced in the preceding section (recall that in Ref. [1] the subtraction operator is simply $t_{p}^{d}$, so that all mass variables are "hard" and play no special role in the subtraction procedure). Fortunately, the combinatoric part of the proof may be taken over without any modification whatever, and we merely summarize the results.

Definitions. Let $U \in \widetilde{F}_{G}, \gamma \in U . \bar{\gamma}(U)$ is defined to be the reduced graph obtained by contracting each $\lambda \in U, \lambda \subset \gamma$, to a point. Each line in $\bar{\gamma}(U)$ is labeled as in (4) in $I_{G}(U)$. We say that $U$ is complete with respect to a hyperplane $H$ in the $4 m$-dimensional space of integration variables $k$ if $G \in U$ and $\forall \gamma \in U$, either all lines $L$ of $\bar{\gamma}(U)$ have $k_{L}^{\gamma}$ constant on $H$, or all lines $L$ of $\bar{\gamma}(U)$ have $k_{L}^{\gamma}$ variable on $H$ (more concisely, either $\bar{\gamma}$ is constant or $\bar{\gamma}$ is variable).

Lemma 1. Let $G$ be a proper diagram, $H$ a hyperplane in the space of integration momenta and $\mathscr{C}_{\mathrm{G}}^{\mathrm{H}}$ the set of $\mathrm{H}$-complete forests of $\mathrm{G}$. Then the normalized integrand $R_{G}$ may be written

where

$$
\begin{aligned}
R_{G} & =\sum_{C \in \mathscr{C}_{G}^{H}} R_{G}(C) \\
R_{G}(C) & =S_{G} \prod_{\gamma \in C}\left(f_{\gamma} S_{\gamma}\right) I_{G}(C),
\end{aligned}
$$

$$
f_{\gamma}=\left\{\begin{array}{rll}
-\tau_{\gamma} & \text { if } & \gamma \notin B(C) \cup\{G\} \\
1-\tau_{\gamma} & \text { if } & \gamma \in B(C) \cup\{G\}
\end{array}\right.
$$


with $B(C)=\{\gamma \in C: \bar{\gamma}(C)$ variable, $\gamma$ a maximal element of $C$ contained in $\lambda \in C, \bar{\lambda}(C)$ constant.

$A$ recursive form of the formula for $R_{G}(C)$ is

where

$$
R_{G}(C)=S_{G}\left(1-\tau_{G}\right) Y_{G}(C)
$$

$$
\begin{aligned}
Y_{\gamma}(C) & =I_{\bar{\gamma}(C)} S_{\gamma} \prod_{i=1}^{a} f_{\gamma_{2}} Y_{\gamma_{i}}(C), \\
\bar{\gamma}(C) & =\gamma / \gamma_{1} \ldots \gamma_{a},
\end{aligned}
$$

$I_{\bar{\gamma}(C)}=$ part of unsubtracted integral corresponding to $\bar{\gamma}(C)$.

The remainder of the power-counting theorem may be stated as follows:

Theorem. Let $G$ be a proper diagram, $H$ a hyperplane in the space of integration momenta $k$ of the corresponding Feynman integral, and $C$ a complete forest of $G$ with respect to $H$. Let $R_{G}(C)$ be the partially subtracted integral defined by (10) or (11) with $\tau_{\gamma}$ a subtraction operator (as defined in Section 3). If $t_{1}, t_{2}, \ldots, t_{h}$ are the independent real parameters of $H$, then

$$
\operatorname{deg}_{t} R_{G}(C)<-h
$$

so that the superficial divergence of the formal integral

$$
\int_{H} R_{G}(C) d^{h} t
$$

is negative. Since $H$ and $C$ where chosen arbitrarily, we conclude, referring to (10), that $J_{G_{\varepsilon}}$ and all of its subintegrals have negative superficial divergence.

Proof. The proof depends crucially on the various properties of the subtraction operator $\tau_{\gamma}$, and we shall refer to these by the appropriate small Roman numerals. We first eastablish (for given $H$ and $C \in \mathscr{C}_{G}^{H}$ ) the following inequalities for $Y_{\gamma}(C) \equiv Y_{\gamma}\left(k(t), p^{\gamma}, w^{\gamma}\right)$ defined by (11):

$$
\begin{aligned}
& \operatorname{deg}_{t p^{\gamma} w^{\gamma}} Y_{\gamma}\left(k(t), p^{\gamma}, w^{\gamma}\right) \leqq d(\gamma)-M(\gamma) \text { if } \bar{\gamma} \text { is variable } \\
& \operatorname{deg}_{t} Y_{\gamma}\left(k(t), p^{\gamma}, w^{\gamma}\right) \leqq-M(\gamma) \text { if } \bar{\gamma} \text { is constant, }
\end{aligned}
$$

equality holding only if $M(\gamma)=0$, where

$$
M(\gamma)=4 \times \sum_{\substack{\mu \in C \\ \mu \subset \gamma, \bar{\mu}}} \text { variable }
$$

(note $M(G) \geqq h=$ dimension of $H$ ).

The proof is by induction. We assume that the equalities hold for all $\lambda \in C, \lambda \subset \gamma$. 
Case I: $\bar{\gamma}$ constant. The result follows from (11) and

a) $\operatorname{deg}_{t} I_{\bar{\gamma}}=0$,

b) $\operatorname{deg}_{t} S_{\gamma} \tau_{\gamma \alpha} Y_{\gamma_{\alpha}} \leqq-M\left(\gamma_{\alpha}\right)$ if $\bar{\gamma}_{\alpha}$ is constant, equality holding only if $M\left(\gamma_{\alpha}\right)=0$.

\section{Proof.}

$\operatorname{deg}_{t} Y_{\gamma_{\alpha}}\left(k(t), p^{\gamma_{\alpha}}, w^{\gamma_{\alpha}}\right) \leqq-M\left(\gamma_{\alpha}\right), \quad$ etc., by induction hypothesis $\Rightarrow \operatorname{deg}_{t} \tau_{\gamma_{\alpha}} Y_{\gamma_{\alpha}}\left(k(t), p^{\gamma_{\alpha}}, w^{\gamma_{\alpha}}\right) \leqq-M\left(\gamma_{\alpha}\right), \quad$ etc., by (vi) $\Rightarrow \operatorname{deg}_{t}\left[S_{\gamma} \tau_{\gamma_{\alpha}} Y_{\gamma_{\alpha}}\right]\left(k(t), p^{\gamma}, w^{\gamma}\right)=\operatorname{deg}_{t} \tau_{\gamma_{\alpha}} Y_{\gamma_{\alpha}}\left(k(t), p^{\gamma_{\alpha}}\left(k(t), p^{\gamma}\right), w^{\gamma}\right) \leqq-M\left(\gamma_{\alpha}\right)$ (= only if $M\left(\gamma_{\alpha}\right)=0$ ) since $p^{\gamma_{\alpha}}\left(k(t), p^{\gamma}\right)$ is independent of $t$ for $\bar{\gamma}$ constant.

c) $\operatorname{deg}_{t} S\left(1-\tau_{\gamma_{\alpha}}\right) Y_{\gamma_{\alpha}}<-M\left(\gamma_{\alpha}\right)$ if $\bar{\gamma}_{\alpha}$ is variable.

\section{Proof.}

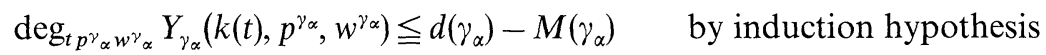

$$
\begin{aligned}
& \operatorname{deg}_{t}\left(1-\tau_{\gamma_{\alpha}}\right) Y_{\gamma_{\alpha}}\left(k(t), p^{\gamma_{\alpha}}, w^{\gamma_{\alpha}}\right)<-M\left(\gamma_{\alpha}\right) \quad \text { by (vii) } \\
& \operatorname{deg}_{t}\left[S_{\gamma}\left(1-\tau_{\gamma_{\alpha}}\right) Y_{\gamma_{\alpha}}\right]\left(k(t), p^{\gamma}, w^{\gamma}\right) \\
& =\operatorname{deg}_{t}\left(1-\tau_{\gamma_{\alpha}}\right) Y_{\gamma_{\alpha}}\left(k(t), p^{\gamma_{\alpha}}\left(k(t), p^{\gamma}\right), w^{\gamma}\right)<-M\left(\gamma_{\alpha}\right) \text {. }
\end{aligned}
$$

Case II : $\bar{\gamma}$ variable. The result follows from (11) and

a) $\operatorname{deg}_{t p^{\gamma} w^{\gamma}} I_{\bar{\gamma}}=d(\bar{\gamma})-M(\bar{\gamma})$,

b) $\operatorname{deg}_{t p^{\gamma} w^{\gamma}} S_{\gamma_{\alpha}} \tau_{\gamma_{\alpha}} Y_{\gamma} \leqq d\left(\gamma_{\alpha}\right)-M\left(\gamma_{\alpha}\right)$ for $\bar{\gamma}_{\alpha}$ variable.

\section{Proof.}

$$
\begin{aligned}
& \operatorname{deg}_{t p^{\gamma}{ }_{\alpha} w_{\alpha}{ }_{\alpha}} Y_{\gamma_{\alpha}}\left(k(t), p^{\gamma_{\alpha}}, w^{\gamma_{\alpha}}\right) \leqq d\left(\gamma_{\alpha}\right)-M\left(\gamma_{\alpha}\right) \quad \text { by induction hypothesis } \\
& \operatorname{deg}_{t p^{\gamma_{\alpha}} w^{\gamma_{\alpha}}} \tau_{\gamma_{\alpha}} Y_{\gamma_{\alpha}}\left(k(t), p^{\gamma_{\alpha}}, w^{\gamma_{\alpha}}\right) \leqq d\left(\gamma_{\alpha}\right)-M\left(\gamma_{\alpha}\right) \text { by (v) } \\
& \operatorname{deg}_{t p^{\gamma} w^{\gamma}}\left[S_{\gamma} \tau_{\gamma_{\alpha}} Y_{\gamma_{\alpha}}\right]\left(k(t), p^{\gamma}, w^{\gamma}\right) \\
& =\operatorname{deg}_{t p^{\gamma} w^{\gamma}} \tau_{\gamma_{\alpha}} Y_{\gamma_{\alpha}}\left(k(t), p^{\gamma_{\alpha}}\left(k(t), p^{\gamma}\right), w^{\gamma}\right) \leqq d\left(\gamma_{\alpha}\right)-M\left(\gamma_{\alpha}\right) \text {. } \\
& \text { c) } \operatorname{deg}_{t p^{\gamma} w^{\gamma}} S_{\gamma} \tau_{\gamma_{\alpha}} Y_{\gamma_{\alpha}} \leqq d\left(\gamma_{\alpha}\right)-M\left(\gamma_{\alpha}\right) \text { for } \bar{\gamma}_{\alpha} \text { constant. }
\end{aligned}
$$

\section{Proof.}

$$
\begin{array}{ll}
\operatorname{deg}_{t} Y_{\gamma_{\alpha}}\left(k(t), p^{\gamma_{\alpha}}, w^{\gamma_{\alpha}}\right) \leqq-M\left(\gamma_{\alpha}\right) & \text { by induction hypothesis } \\
\operatorname{deg}_{t} \tau_{\gamma_{\alpha}} Y_{\gamma_{\alpha}}\left(k(t), p^{\gamma_{\alpha}}, w^{\gamma_{\alpha}}\right) \leqq-M\left(\gamma_{\alpha}\right) & \text { by (vi) } \\
\operatorname{deg}_{p^{\gamma_{\alpha}} w_{\gamma_{\alpha}} \tau_{\gamma_{\alpha}} Y_{\gamma_{\alpha}}\left(k(t), p^{\gamma_{\alpha}}, w^{\gamma_{\alpha}}\right) \leqq d\left(\gamma_{\alpha}\right)} & \text { by (iii) }
\end{array}
$$


Hence

$$
\begin{aligned}
& \operatorname{deg}_{t p^{\gamma} w^{\gamma}{ }_{\alpha}} \tau_{\gamma_{\alpha}} Y_{\gamma_{\alpha}}\left(k(t), p^{\gamma_{\alpha}}, w^{\gamma_{\alpha}}\right) \leqq d\left(\gamma_{\alpha}\right)-M\left(\gamma_{\alpha}\right) \\
& \operatorname{deg}_{t p^{\gamma} w^{\gamma}}\left[S_{\gamma} \tau_{\gamma_{\alpha}} Y_{\gamma_{\alpha}}\right]\left(k(t), p^{\gamma}, w^{\gamma}\right) \\
= & \operatorname{deg}_{t p^{\gamma} w^{\gamma}} \tau_{\gamma_{\alpha}} Y_{\gamma_{\alpha}}\left(k(t), p^{\gamma_{\alpha}}\left(k(t), p^{\gamma}\right), w^{\gamma}\right) \leqq d\left(\gamma_{\alpha}\right)-M\left(\gamma_{\alpha}\right) .
\end{aligned}
$$

To complete the proof of the theorem, we apply these inequalities to $G$ itself.

Case I: $\bar{G}$ constant. Since $M(G) \geqq h \neq 0$, we have $\operatorname{deg}_{t} Y_{G}<-M(G)$. Then $\operatorname{deg}_{t} R_{G}(C)=\operatorname{deg}_{t}\left(1-\tau_{G}\right) Y_{G}<-M(G) \leqq-h$ by (vi).

Case II: $\bar{G}$ variable. Then $\operatorname{deg}_{t p w} Y_{G} \leqq d(G)-M(G)$. Hence $\operatorname{deg}_{t} R_{G}(C)$ $=\operatorname{deg}_{t}\left(1-\tau_{G}\right) Y_{G}<-M(G) \leqq-h$ by (vii).

This completes the proof of the theorem. If the mass parameters of the theory are such that $\mu_{a b \sigma}^{2}$ in Eq. (4) is strictly positive for every line $L_{a b \sigma}$, then the absolute convergence of $J_{G_{\varepsilon}}$ and the existence of $J_{G}=\lim _{\varepsilon \rightarrow 0} J_{G} \varepsilon$ as a Lorentz-invariant tempered distribution follows immediately from the theorems of Ref. [1]. If some of the $\mu_{a b \sigma}^{2}$ vanish, the theorem of this section remains valid, but does not imply the absolute convergence of $J_{G \varepsilon}$.

\section{Applications}

We now indicate how the modified subtraction procedure introduced above can be used to formulate perturbative models in which terms of the unperturbed Lagrangian are assigned degrees less than four. In general we may write

$$
\mathscr{L}_{\mathrm{EFF}}=\sum_{i=1}^{N} c_{i}\left\{M_{i}\right\}_{\delta_{i}}
$$

where $M_{i}$ is a product of basic fields $A_{j}$ and their derivatives, $\delta_{i}, 2 \leqq \delta_{i} \leqq 4$, is the subtraction degree assigned to $M_{i}$, and $c_{i}$ is proportional to a product of masses and a power of the perturbation parameter (assumed dimensionless without loss of generality).

To construct the renormalized Green functions of any number of basic and composite (normal-product) fields, we introduce

and define

$$
\mathscr{L}_{s}=\sum_{i=1}^{N} c_{i} s^{4-\delta_{i}} M_{i}=\mathscr{L}_{0 s}+\mathscr{L}_{I s} \quad(0 \leqq s \leqq 1)
$$

$$
\begin{aligned}
& \left\langle T \prod_{j} A_{j}\left(x_{j}\right) \prod_{k} N_{\eta_{k}}\left[Q_{k}\left(y_{k}\right)\right]\right\rangle \\
& =\text { Finite part } \frac{\left\langle 0\left|T \prod_{j} A_{j}^{(0 s)}\left(x_{j}\right) \prod_{k} Q_{k}^{(0 s)}\left(y_{k}\right) \exp \left[i \int \mathscr{L}_{I s}^{(0 s)} d x\right]\right| 0\right\rangle}{\left\langle 0\left|\exp \left[i \int \mathscr{L}_{I s}^{(0 s)}(x) d x\right]\right| 0\right\rangle}
\end{aligned}
$$


where $A_{j}^{(0 s)}$ is a free field whose two-point function is specified by $\mathscr{L}_{0 s}$. The righthand side corresponds to the usual sort of Feynman diagram expansion (note, however, that $\mathscr{L}_{I s}^{(0)}$ and $Q_{k}^{(0 s)}$ are not Wick-ordered, so that there will be diagrams in which a line both begins and ends at the same vertex). For a given proper diagram $G$, the unsubtracted integrand is of the general form (2), and we may apply the subtraction formula (3), with

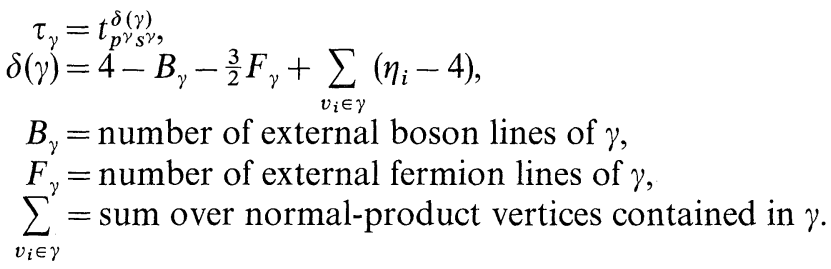

To apply the power-counting theorem of Section 4, we note that each coefficient $c_{i}$ in (14) may be written as a (non-unique) product of "hard" and "soft" mass parameters, multiplied by a dimensionless quantity:

$$
\begin{gathered}
c_{i}=d_{i} g^{n_{i}} m_{i 1} \ldots m_{i \alpha(i)} w_{i 1} \ldots w_{i \beta(i)}, \\
\alpha(i)=\delta_{i}-\operatorname{dim} M_{i}, \quad \beta(i)=4-\delta_{i} .
\end{gathered}
$$

From (14) and (16), we see that we may write

$$
\begin{gathered}
I_{G}=I_{G}(p, k, m, s w) \\
m=\left\{m_{i j}: i=1, \ldots, N ; j=1, \ldots, \alpha(i)\right\}, s w=\left\{s w_{i j}: i=1, \ldots, N ; j=1, \ldots \beta(i)\right\},
\end{gathered}
$$

so that it is obvious that in constructing $R_{G}$ it makes no difference whether one uses $\tau_{\gamma}=t_{p^{\gamma} s^{\gamma}}^{\delta(\gamma)}$ or $\tau_{\gamma}=t_{p^{\gamma} w^{\gamma}}^{\delta(\gamma)}$. With the latter choice, the theorem is directly applicable. Thus the Green functions (15) are well defined, Lorentz invariant tempered distributions at least if all propagators have non-vanishing masses).

It is not obvious that the Green functions constructed in the above manner are consistent with the original interpretation of $\delta_{i}$ in Eq. (13). More precisely, do the Green functions defined by (15) satisfy the field equations (in the sense of normal products)

$$
\begin{aligned}
& \left\langle T\left[\frac{\partial \mathscr{L}_{\mathrm{EFF}}}{\partial A_{j}}(z)-\partial^{\mu} \frac{\partial \mathscr{L}_{\mathrm{EFF}}}{\partial\left(\partial^{\mu} A_{j}\right)}(z)\right] \prod_{l} A_{i_{l}}\left(x_{l}\right)\right\rangle \\
& =i \sum_{k} \delta_{j k} \delta\left(z-x_{k}\right)\left\langle T \prod_{k \neq j} A_{i_{k}}\left(x_{k}\right)\right\rangle, \\
& \left\langle T A_{m}(z)\left[\frac{\partial \mathscr{L}_{\mathrm{EFF}}}{\partial A_{j}}(z)-\partial^{\mu} \frac{\partial \mathscr{L}_{\mathrm{EFF}}}{\partial\left(\partial^{\mu} A_{j}\right)}(z)\right] \prod_{l} A_{i_{l}}\left(x_{l}\right)\right\rangle \\
& =i \sum_{k} \delta_{j k} \delta\left(z-x_{k}\right)\left\langle T A_{m}(z) \prod_{k \neq j} A_{i_{k}}\left(x_{k}\right)\right\rangle ?
\end{aligned}
$$


That this is indeed the case is an easy exercice (strictly analogous to the derivation in the case where $\mathscr{L}$ contains only terms of subtraction degree four [5]) which we omit.

For a more detailed description of various recent applications of the modified subtraction procedure, the reader may wish to consult Refs. $[7-10]$.

\section{References}

1. Zimmermann, W.: Commun. math. Phys. 15, 208 (1969)

2. Zimmermann, W.: Commun. math. Phys. 11, 1 (1969)

3. Bogoliubov, N. N., Shirkov, D.V.: Introduction to the Theory of Quantized Fields. New York: Interscience 1959

4. Hepp, K.: Commun. math. Phys. 2, 301 (1966)

5. Lowenstein, J.H.: Phys. Rev. D4, 2281 (1971)

6. Gomes, M., Lowenstein, J. H.: Phys. Rev. D7, 550 (1973)

7. Jegerlehner, F., Schroer, B. : Nucl Phys. B 68, 461 (1974)

8. Lowenstein,J.H., Schroer,B., Weinstein, M., Zimmermann,W.: SLAC and NYU reports, 1974

9. Lowenstein, J.H., Rouet, A., Stora, R., Zimmermann, W.: Lectures at Capri Summer School, 1973, C.N.R.S. Marseille report

10. Gomes, M., Schroer, B.: São Paulo report, 1974

Communicated by K. Symanzik

J. H. Lowenstein Department of Physics New York University 4, Washington Place New York, N. Y. 10003, USA 